\title{
First principles studies of the effect of nickel carbide catalyst composition on carbon nanotube growth
}

\author{
Anders Börjesson* \\ University of Borås, 50190 Borås, Sweden \\ University of Gothenburg, Gothenburg, Sweden \\ Kim Bolton \\ University of Borås, Borås, Sweden
}

(Dated: July 23, 2010)

\begin{abstract}
Density functional theory calculations were used to investigate the stability of single walled carbon nanotubes (CNTs) attached to nanoparticles. The total energies and the adhesion energies between the CNTs and the nanoparticles were calculated for systems where the nanoparticles were either pure Ni or Ni carbide. It was found that the adhesion between the CNT and a pure Ni cluster is stronger than between the same CNT and a Ni-carbide cluster although the energy difference was small compared to the total adhesion energies. This adhesion strength implies that CNTs are likely to remain attached to both pure $\mathrm{Ni}$ and Ni-carbide clusters and that either pure $\mathrm{Ni}$ or Ni-carbide clusters may be docked onto the open CNT ends to achieve continued growth or electronic contacts between CNTs and electrode materials. The system with a CNT attached to a pure Ni cluster was found to be energetically favoured compared to a system containing the same CNT attached to a Ni carbide. The difference in total energy implies that a CNT should act as a sink for $\mathrm{C}$ atoms dissolved in the Ni-carbide cluster which means that the dissolved $\mathrm{C}$ atoms will be drained from the cluster, yielding a pure metal in the zero Kelvin thermodynamic limit. It is argued that this draining procedure is likely to occur even if carbon is added to the cluster at a proper rate, e.g. during CNT growth.
\end{abstract}


In order to realize the high expectations of carbon nanotubes (CNTs) within the field of nano electronics[1, 2] a procedure for systematic production of CNTs with predetermined chirality is desirable. During the past two decades this has been achieved with some degree of success, either by selective growth[3-5] or by separation of CNTs $[6-8]$.

A logical step in the search for understanding CNT growth is to investigate the importance of the individual parameters, e.g, growth mechanisms[9-14], catalyst particles[15-17] and substrates[18-21] that might affect the final product.

In addition to experimental work, valuable information has been obtained by computational studies, spanning from first principles density functional theory (DFT) [22-30], via semi-empirical methods [31-34] to entirely empirical methods[35-37].

Another issue which still remains to be resolved before the realization of large scale production of nano electronics is the construction and properties of the electronic junctions between CNTs and any contact material. The electronic transport properties though SWNTbased devices depends on the SWNT chirality as well as on the structure and bonding at the electrode-SWNT interface[38]. Hence, it can be expected that the conductive properties will differ widely depending on the manufacturing process.

This work aims at increasing the understanding of how the constitution of the catalyst particle may affect the CNT growth as well as the contacts produced by the docking of metal particles to existing CNTs. This intriguing question has been addressed by first principles DFT calculations of the total and adhesion energies of CNTs attached to Ni (carbide) particles.

The majority of the DFT calculations presented in this contribution were performed using the Vienna ab initio simulation package (VASP)[39] using a plane-wave basis set in combination with ultrasoft pseudo potentials (US-PP). The numerical results are from spin polarised calculations performed using the PW91[40] exchange-correlation functional with a plane wave energy cut-off of $400 \mathrm{eV}$ which converged the total energies to less than $1 \mathrm{meV} /$ atom. The atomic structures were positioned in a simulation box of size $15 \AA \times 15 \AA \times 30 \AA$, which was sufficiently large to allow for $\Gamma$-point sampling of $k$-space. The geometry optimisations of the atomic systerms, performed using the conjugate gradient algorithm, were stopped when the total energy difference between subsequent relaxation steps was less than $10^{-4}$ $\mathrm{eV}$. This method and parameters have been successfully used in previous studies of CNTmetal systems, e.g., in Refs. [29, 30, 33, 41]. In order to verify the results obtained by 
VASP, a few control calculations were performed using the DFT program GPAW[42] with the PBE[43] exchange-correlation functional. The GPAW calculations were performed using the projector augmented wave method on a real space grid. The grid spacing was less than $0.25 \AA$ and the box size was large enough to allow for at least $5 \AA$ vacuum between the atomic system and the box edges. The relaxed structures obtained from VASP were used as input structures for relaxation with the quasi-Newton method implemented in the ASE[44] package and with forces calculated by GPAW with the above mentioned parameters. These control calculations showed similar trends and the results are presented for comparison.

It is worth mentioning that the total energies presented in this work are relative to reference states, defined either by the US-PP (VASP calculations) or PAW setups (GPAW calculations). The consequence of this is that the total energies obtained by the two different methods are not directly comparable since the references are different. This difference does not affect the conclusions presented in this contribution since these are solely based on energy differences within each method.

All atomic configurations have a CNT with one end attached to a cluster particle, being either pure $\mathrm{Ni}$ or a Ni-carbide, and the other end passivated, either by a cap or a ring of $\mathrm{H}$ atoms. Four different CNT chiralities, $(3,3),(5,5),(9,1)$ and $(10,0)$, were used in the study. All CNTs had approximate lengths between 5.4 to $8.5 \AA$ which were expected to be sufficiently long to ensure that the qualitative properties studied close to the open end are not incriminated by the vicinity of another, passivated end. The quantitative properties may however be slightly affected by the CNT length but that is not likely to be important here since the conclusions are based on observation of energy differences in the order eV. All systems with a certain CNT chirality contained the same number of $\mathrm{Ni}$ and $\mathrm{C}$ atoms, meaning that the CNTs attached to pure Ni were slightly longer than CNTs attached to Ni-carbide.

The pure Ni clusters had an icosahedral shape with the exception for a reference system containing an amorphous (non-icosahedral) $\mathrm{Ni}_{55}$ cluster, used to identify possible effects due to the icosahedral geometry of the cluster. The choice of the $\mathrm{Ni}_{55}$ cluster size was motivated by experimental reports of CNT growth from catalysts whose size are similar to or slightly larger than the CNT diameters[45, 46]. The choice was also motivate by the use of the same system in other studies, e.g., in Refs. [30, 33, 41]

The carbide clusters were amorphous and the $\mathrm{C}$ concentrations within the clusters were 
$6 \mathrm{C}$ atoms for the $(3,3) \mathrm{CNT}$ and $20 \mathrm{C}$ atoms for the $(5,5),(9,1)$ and $(10,0) \mathrm{CNTs}$, which corresponded to the removal of 6 and $20 \mathrm{C}$ atoms from the longer CNTs. In addition to the removal of 20 atoms, the $(9,1) \mathrm{CNT}$ allowed for a consecutive removal of $\mathrm{C}$ dimers without compromising the structure of the CNT end. The additional $\mathrm{C}$ concentrations studied were 6 and $14 \mathrm{C}$ atoms dissolved into the $\mathrm{Ni}$ particle. The results for the carbide clusters originate from structures where $\mathrm{C}$ atoms in the cluster may be attached to the CNT structure but no new polygons were formed in association with the CNT.

Since the amorphous and carbide clusters had very complicated geometries, a few different starting configurations were created for most of these systems and the results presented in this contribution originate from the most stable geometry. Despite this caution, it should be noted that the energies of the relaxed structures may still be sensitive to the starting conditions. Hence, one has to be very careful when drawing conclusions based on only one set of calculations, e.g., for only one chirality, and conclusions based on small energy differences.

Additional details about the preparation of the CNT-cluster systems is given in the Supporting Information. Examples of typical minimum energy structures of $(5,5)$ and $(10,0)$ CNTs attached to pure $\mathrm{Ni}$ and Ni-carbide clusters are seen in Fig. 1.

In the discussion belo, a CNT containing $X \mathrm{C}$ atoms attached to a cluster containing $Y \mathrm{Ni}$ atoms and $Z \mathrm{C}$ atoms will be denoted $\mathrm{CNT}_{X} \mathrm{Ni}_{Y} \mathrm{C}_{Z}$. For example, a CNT containing $66 \mathrm{C}$ atoms attached to a cluster containing $55 \mathrm{Ni}$ atoms and $14 \mathrm{C}$ atoms is denoted $\mathrm{CNT}_{66} \mathrm{Ni}_{55} \mathrm{C}_{14}$.

The adhesion, or binding, energies between the CNT structure and Ni(carbide) have been calculated as

$$
E_{\text {binding }}=E_{C N T_{X} N i_{Y} C_{Z}}-E_{C N T_{X}}-E_{N i_{Y} C_{Z}}
$$

where $E_{C N T_{X} N i_{Y} C_{Z}}$ is the energy of the total system while $E_{C N T_{X}}$ and $E_{N i_{Y} C_{Z}}$ are the energies of the CNT and cluster, respectively.

The energies presented in this contribution were obtained from the lowest energy geometries unless otherwise stated, i.e., the energies are for relaxed atomic configurations. For example, in the calculation of the adhesion energies, the $\mathrm{CNT}_{X} \mathrm{Ni}_{Y} \mathrm{C}_{Z}, \mathrm{CNT}_{X}$ and $\mathrm{Ni}_{Y} \mathrm{C}_{Z}$ systems had been relaxed individually. In addition to this, a few direct adhesion energies were calculated by a direct separation of the $\mathrm{CNT}_{X} \mathrm{Ni}_{Y} \mathrm{C}_{Z}$ structure, i.e., the $\mathrm{CNT}_{X}$ and $\mathrm{Ni}_{Y} \mathrm{C}_{Z}$ systems had not been relaxed individually after the separation. When the direct adhesion energies are discussed this is explicitly clarified. 
The work presented in this contribution expands and advances the work presented in Ref. [33]. Briefly, the previous work focussed on the junction between a CNT and a Ni cluster and the calculations performed showed that the CNT is attached above the metal surface (and not at subsurface sites) and that the metal is more prone to adapt to the CNT-structure and not vice versa. The present contribution shows, among other things, that the same behaviour is observed for CNTs attached to Ni carbides, as illustrated in Fig. 1.

The total energies presented in Table I show that the $(10,0)$ nanotube is energetically favoured compared to the other chiralities when it is attached to a pure Ni cluster. This is in agreement with the results presented in Ref. [30] and it could therefore be reasonable to believe that this is the case for the CNT attachment to carbides as well. While this may be indicated by the results in Table I, the harsh energy landscape makes the results more dependent on initial configuration, i.e., this can not be concluded with certainty.

From the results in Table I, it is also seen that adhesion energy between pure Ni and the zig-zag $(10,0) \mathrm{CNT}$ is almost $10 \mathrm{eV}$ stronger than for the archair $(5,5) \mathrm{CNT}$, i.e., $E_{a d,(10,0)-N i_{55}}=-25.20 \mathrm{eV}$ compared to $E_{a d,(5,5)-N i_{55}}=-16.22 \mathrm{eV}$. Separation of the $\mathrm{Ni}_{55}$ and the CNT generates 10 dangling $\mathrm{C}$ bonds, independently of whether the CNT has chirality $(10,0)$ or $(5,5)$, which results in adhesion energies of approximately $-2.5 \mathrm{eV}$ and $-1.6 \mathrm{eV}$ per dangling bond, respectively. This is in very good agreement with the data presented for $\mathrm{Ni}$ in Fig. 2 of Ref. [30].

Table I also shows that, similarly to the situation for the pure Ni cluster, the adhesion between the $(10,0) \mathrm{CNT}$ and the carbide cluster is stronger than between the $(5,5) \mathrm{CNT}$ and the carbide cluster, but the difference in adhesion energies is slightly reduced. The (absolute values of the) adhesion energies have also decreased to $E_{a d,(10,0)-N i_{55} C_{20}}=-21.30$ $\mathrm{eV}$ and $E_{a d,(5,5)-N i_{55} C_{20}}=-13.90 \mathrm{eV}$.

The direct adhesion energies between the CNT and Ni carbide clusters were also calculated by a direct separation, i.e. where the CNT and cluster had not been relaxed after separation. This was done for the $(5,5),(9,1)$, and $(10,0)$ CNTs attached to $\mathrm{Ni}_{55} \mathrm{C}_{20}$ clusters and the results are presented in Table II where the direct adhesion energies are seen to be $24.07 \mathrm{eV}, 29.83 \mathrm{eV}$ and $30.20 \mathrm{eV}$ for the $(5,5),(9,1)$, and $(10,0)$ CNTs respectively. The direct adhesion energies show the same trend as the adhesion energies obtained after individual relaxation, i.e., the adhesion between a $(10,0)$ CNT and a metal carbide is stronger than the adhesion between a $(5,5) \mathrm{CNT}$ and a metal carbide. The total energies of both the 
CNT and cluster structures obtained after direct separation are seen to be a few eV higher in energy than energies obtained for the same structure after relaxation. The higher energies of the $(9,1)-\mathrm{Ni}_{55} \mathrm{C}_{20}$ and the corresponding $\mathrm{Ni}_{55} \mathrm{C}_{20}$ compared to the energies of the other structures indicate that the energies of these structures may have an effect of the starting configuration. While this affect the total energy, it is not likely to affect the adhesion energy as the cluster is present in both cases.

These results indicate that the geometry of both the CNT and the cluster are affected by the attachment to eachother. In agreement with the results presented in Ref. [33], the geometry of the CNT end retain its qualitative shape after the attachment. The few eV energy difference between the CNT obtained after separation and the relaxed CNT are to a high extent an effect that is not explained by a visible geometrical change, although the $(9,1)$, and $(10,0)$ CNTs showed some tendency to decrease the diameter of the lowest CNT ring. This could be interpreted as the first step towards the formation of a fullerene instead of a open ended CNT.

In Ref. [30] is was argued that a too strong C-metal adhesion is likely to lead to encapsulation of the catalyst, and hence an abortion of the growth process, while a too weak C-metal adhesion is more likely to lead to a detachment of the cluster particle from the $\mathrm{CNT}$ and no further growth. An intermediate adhesion strength is required for continued growth. In this perspective the results presented here indicate that a CNT, initially grown from a pure metal cluster, will remain attached to the cluster even if large amount of $\mathrm{C}$ becomes dissolved in the cluster or, vice versa, a CNT, initially grown from a carbide catalyst particle, will remain attached to the catalyst even if it is drained of carbon. The small difference in adhesion energy is not likely to change the premises of the growth, i.e., the adhesion strength is not likely to be neither to strong nor to weak after dissolution.

The significant adhesion strengths between CNTs and both pure Ni and Ni carbide clusters are also of importance for CNT-electrode contacts as it show that CNT-Ni (carbide) contacts are thermodynamically favourable compared to separation. Although the conductive properties are likely to differ for the pure metal or carbide, the adhesion seen here offers the possibility of electron transfer across the CNT - metal(carbide) interface.

From a comparison of the total energies of CNTs attached to a pure metal (top in Table I) or to carbide (bottom in Table I) it is seen that the CNTs attached to Ni carbides have from $\approx 2 \mathrm{eV}$ (for $(3,3))$ up to $\approx 10 \mathrm{eV}$ (for $(10,0)$ ) higher total energy than the CNTs attached 
to a pure metal clusters. The consequence of this trend, which is observed for all systems, is that under equilibrium conditions, the CNT will act as a sink for $\mathrm{C}$ atoms available in the cluster. Hence, if a CNT is grown from a metal carbide[47] the growth process will not only be aborted when the addition of the external feedstock is stopped but the Ni carbide will be drained of $\mathrm{C}$ atoms and a pure $\mathrm{Ni}$ cluster will remain at the CNT end. This is of relevance to CNT growth, regrowth and CNT-electrode contacts where excess dissolved carbon atoms will diffuse from the metal to the CNT. These cluster particles can either be carbides or become carbides as an effect of the docking procedure, e.g., the procedure referred to as forced docking in Ref. [33]. By careful adjustment of external parameters, such as temperature, pressure and the surrounding atmosphere, the cluster particle can be purified by an annealing process and finally result in a pure CNT-metal junction. Another important consequence of this result is that the formation of a carbide, with moderate carbon concentration, will not necessarily terminate the growth process. Note that this conclusion pertains to the nano-sized clusters studied here, and may not be relevant to bulk metal(carbide) electrodes

The differences in the total energy of systems with pure $\mathrm{Ni}$ and $\mathrm{Ni}$ carbide can, at least to some extent, be explained by the fact that the pure metal clusters are in high symmetry (icosahedral) structures while the metal carbide clusters are amorphous. This effect could be reduced by considering pure metal clusters initially having amorphous, non-crystalline geometries. To verify the validity of the results presented above, a reference model with a $(9,1)$ CNT attached to an amorphous $\mathrm{Ni}_{55}$ cluster, seen in Fig. 2, was compared to the same CNT attached to the icosahedral $\mathrm{Ni}_{55}$ cluster The total energy of the system with the amorphous cluster is $-965.76 \mathrm{eV}$ which is approximately $3 \mathrm{eV}$ higher than the total energy of the system where the same CNT is attached to the icosahedral cluster. The total energy of the $\mathrm{Ni}_{55}$ cluster is increased from $-250,61 \mathrm{eV}$ for the icosahedral cluster to $-248,01 \mathrm{eV}$ for the amorphous cluster. This leads to an adhesion energy between the amorphous $\mathrm{Ni}_{55}$ cluster and the $(9,1)$ CNT of approximately $-22.3 \mathrm{eV}$ which is slightly weaker, although still comparable, to the adhesion between the $(9,1)$ CNT and an icosahedral $\mathrm{Ni}_{55}$.

This leads to the conclusion that the higher energy of the carbide systems is likely to be an effect of both the destruction of the icosahedral structure and the $\mathrm{C}$ dissolution into the cluster. Since the adhesion between the CNT and pure Ni clusters appears to be more or less independent of the structure of the $\mathrm{Ni}$ cluster, it is likely that the reduction in adhesion 
strength between CNTs and carbide clusters, as compared to CNTs and pure Ni clusters is an effect of the $\mathrm{C}$ dissolution. This reduction in the adhesion strength does also conribute to the difference in the total energies calculated for the pure $\mathrm{Ni}$ and $\mathrm{Ni}$ carbide systems.

The two situations discussed so far can be considered as two extremes, i.e., the clusters contain either no or many $\mathrm{C}$ atoms. To ensure that the conclusions drawn are not sensitive to the $\mathrm{C}$ concentrations, calculations were performed for a $(9,1)$ CNT attached to a Ni cluster with $0,6,14$ and 20 dissolved $\mathrm{C}$ atoms. The final structures and total energies are seen in Fig. 3. The total energies (round symbols in Fig. 4) are seen to increase with increasing $\mathrm{C}$ concentration. Also the adhesion energies (squares in Fig. 4) between the CNTs and metal (carbide) are seen to increase with increasing $\mathrm{C}$ concentration, i.e., the adhesion strength becomes weaker with increasing $\mathrm{C}$ concentration. To be able to extract some quantitative trends about the increase in the energy, a linear function (solid line in Fig. 4) was fitted to the data of the total energy as a function of the number of dissolved carbon atoms in the cluster. The total energy per additional $\mathrm{C}$ atom is approximately $0.42 \mathrm{eV} /$ atom. Also the adhesion energy is seen to increase with increasing $\mathrm{C}$ concentration. This increase is very limited when dissolving only $6 \mathrm{C}$ atoms which may be explained that very few dissolved $\mathrm{C}$ atoms are, as seen in Fig. 3 (d), positioned in the part of the cluster that is attached to the CNT. These results are agreement with the idea that a part of the increase in total energy is due to the increase in adhesion energy while the other part of the energy increase must be due to decreased stability of the cluster and CNT.

It should be stressed that the linear dependence is not properly investigated but merely introduced as a tool for the discussion. It is not reasonable that the total energies will show such linear dependence for higher carbon concentrations. Despite the fact that the linear dependence of total and adhesion energies is somewhat artificial, it strongly supports the conclusion that the system is more stable when all carbon atoms are associated with the CNT. The results reported here for the $(9,1)$ CNT are expected to be valid for the other CNTs studied here.

The results discussed above were obtained in the $0 \mathrm{~K}$ limit while the temperature interval between room temperature and approximately $1000 \mathrm{~K}$ is more relevant to the experimental situations. At these temperatures, vibrations of the atoms may affect the stability of the atomic systems. In order to elucidate this effect, two $(9,1)$ CNTs attached Ni and Ni carbide clusters were subjected to short MD simulations. The starting configurations for the MD 
simulation were prepared from the $(9,1)-\mathrm{Ni}_{55} \mathrm{C}_{0}$ and $(9,1)-\mathrm{Ni}_{55} \mathrm{C}_{20}$ structures providing data for Table I and which are seen in Fig. 3. In order to raise the temperature in the beginning of the simulation, each structure was slightly distorted by a very small random displacement of all atoms before initiation of the MD simulation. The simulation temperatures were then controlled by a thermostat set to $1500 \mathrm{~K}$ but due to the limited simulation time, the temperature of the systems were more likely to be around $800 \mathrm{~K}$ during simulation. This temperature showed to be sufficient to induce both vibrations and structural changes of the simulated systems. The time step of the simulation was $1.5 \mathrm{fs}$ and during the simulation, atomic configurations were sampled after times $300 \mathrm{fs}, 600 \mathrm{fs}, 900 \mathrm{fs}$ and $1200 \mathrm{fs}$. The total energies of these configurations were calculated as well as the direct adhesion energies between the CNT and cluster, i.e., the adhesion energies obtained by direct separation of the structures without any individual optimisation of the CNT and cluster.. To make the MD simulation feasible, the plane wave energy cut-off was lowered to $250 \mathrm{eV}$ during the MD simulation while the total energies and direct adhesion energies presented were obtained from calculations with a plane wave energy cut-off of $400 \mathrm{eV}$.

The results of these simulations are presented in Table III and it is clear that the direct adhesion energies between CNTs and both pure Ni and Ni-carbides are in the order of 25 $30 \mathrm{eV}$ even after the introduction of temperature effects. From the total energies, it can also be seen that the systems with pure Ni clusters are energetically favoured by approximately $8-10 \mathrm{eV}$. This means that, similarly to the $0 \mathrm{~K}$ situation described above, the CNT will act as a sink for $\mathrm{C}$ atoms dissolved into the cluster. The adhesion energy between the CNT and cluster is strong for both the pure $\mathrm{Ni}$ cluster and the Ni-carbide cluster which means that both clusters are likely to remain attached to the CNT. However, the trend of the slightly weaker adhesion between CNTs and Ni-carbide compared to pure Ni was not seen at the finite temperature. This may be due to the fact that such small energy differences may be drowned in the large total energy oscillations at these temperatures, i.e., it could only be seen by averaging over a larger system ensemble. These results indicate that the results obtained for $0 \mathrm{~K}$ situation are likely to be valid even a finite temperatures.

As discussed above, carbon atoms that are dissolved in the cluster are drained into the attached CNT, which acts as a sink. When this occurs there is also an increase in the CNT-cluster adhesion energy. At low carbon feedstock pressure, draining leads to a slightly stronger adhesion and a decrease in total energy. For the maximum allowed feedstock 
pressure, defined such that the addition rate of one $\mathrm{C}$ atom to the system is equal to the rate of $\mathrm{C}$ incorporation into the $\mathrm{CNT}$, the carbon concentration in the cluster particle will be constant. Under this condition the draining process is driven by the decrease total energy and the adhesion energy will remain constant. Exceeding the maximum pressure will mean that the rate of $\mathrm{C}$ addition to the system is faster than the incorporation in the CNT and the consequence of this is not addressed in the present calculations.

First principles DFT calculations, obtained by two different softwares and exchangecorrelation functionals, have shown that a CNT attached to a pure Ni cluster is energetically favoured compared to the same CNT attached to a Ni carbide. It is also seen that the binding (adhesion) strength between the CNT and the Ni carbide cluster is slightly weaker than the binding strength between the CNT and the pure Ni cluster. However, the difference in binding energy is small compared to the total binding energies. This indicates that if the adhesion energy is strong enough for the CNT to remain attached to the pure Ni particle, it will most likely remain attached to the Ni carbide as well. Furthermore, from the difference in total energies the conclusion is drawn that once the CNT is formed on, or mechanically attached to, a Ni particle it will serve as a sink and drain carbon atoms from the Ni particle. These results were obtained at $0 \mathrm{~K}$ but a few control calculations at finite temperature indicate the results should be valid at higher temperatures as well.

We thank Dr. Christophe Bichara and Dr. Hakim Amara for fruitful discussions. The authors are grateful for time allocated on the Swedish National Supercomputing facilities and for support obtained from the C3SE, NSC and UPPMAX computing resources. Financial support was obtained from the Swedish Research Council and the Nanoparticle Platform at University of Gothenburg.

Supporting Information Available: Detailed descriptions of initial atomic structures. This material is available free of charge via the Internet at http://pubs.acs.org.

* anders.borjesson@hb.se

[1] M. P. Anantram and F. Leonard, Rep. Prog. Phys. 69, 507 (2006).

[2] P. Avouris, Z. Chen, and V. Perebeinos, Nature Nanotechnology 2, 605 (2007).

[3] S. M. Bachilo, L. Balzano, J. E. Herrera, F. Pompeo, D. E. Resasco, and R. B. Weisman, J. 
Am. Chem. Soc. 125, 11186 (2003).

[4] G. Lolli, L. Zhang, L. Balzano, N. Sakulchaicharoen, Y. Tan, and D. E. Resasco, J. Phys. Chem. B 110, 2108 (2006).

[5] X. Li, X. Tu, S. Zaric, K. Welsher, W. S. Seo, W. Zhao, and H. Dai, J. Am. Chem. Soc. 129, 15770 (2007).

[6] R. Krupke, F. Hennrich, H. V. Lohneysen, and M. M. Kappes, Science 301, 344 (2003).

[7] M. Zheng, A. Jagota, E. D. Semke, B. A. Diner, R. S. Mclean, S. R. Lustig, R. E. Richardson, and N. G. Tassi, Nature Materials 2, 338 (2003).

[8] Z. Chen, X. Du, M.-H. Du, C. D. Rancken, H.-P. Cheng, and A. G. Rinzler, Nano Letters 3, $1245(2003)$.

[9] J. Kong, H. T. Soh, A. M. Cassell, C. F. Quate, and H. Dai, Nature 395, 878 (1998).

[10] H. Kanzow and A. Ding, Physical Review B 60, 11180 (1999).

[11] K. Bladh, L. K. L. Falk, and F. Rohmund, Appl. Phys. A 70, 317 (2000).

[12] C. Ducati, I. Alexandrou, M. Chhowalla, G. A. J. Amaratunga, and J. Robertson, Journal of Applied Physics 92, 3299 (2002).

[13] D. B. Geohegan, A. A. Puretzky, I. N. Ivanov, S. Jesse, G. Eres, and J. Y. Howe, Applied Physics Letters 83, 1851 (2003).

[14] A. Moisala, A. G. Nasibulin, and E. I. Kauppinen, Journal of Physics: Condensed Matter 15, S3011 (2003).

[15] D. Takagi, Y. Homma, H. Hibino, S. Suzuki, and Y. Kobayashi, Nano Letters 6, 2642 (2006).

[16] W. Zhou, Z. Han, J. Wang, Y. Zhang, Z. Jin, X. Sun, Y. Zhang, C. Yan, and Y. Li, Nano Letters 6, 2987 (2006).

[17] A. R. Harutyunyan, T. Tokune, and E. Mora, Applied Physics Letters 87, 051919 (2005).

[18] P. Buffat and J.-P. Borel, Physical Review A 13, 2287 (1976).

[19] T. Castro, R. Reifenberger, E. Choi, and R. P. Andres, Physical Review B 42, 8548 (1990).

[20] S. L. Lai, J. Y. Guo, V. Petrova, G. Ramanath, and L. H. Allen, Physical Review Letters 77, 99 (1996).

[21] F. Ding, A. Rosén, S. Curtarolo, and K. Bolton, Applied Physics Letters 88, 133110 (2006).

[22] J.-Y. Raty, F. Gygi, and G. Galli, Physical Review Letters 95, 096103 (2005).

[23] J. Gavillet, A. Loiseau, C. Journet, F. Williame, F. Ducastelle, and J.-C. Charlier, Physical Review Letters 87, 275504 (2001). 
[24] F. Ablid-Pedersen, J. K. Nørskow, J. R. Rostrup-Nielsen, J. Sehested, and S. Helveg, Physical Review B 73, 115419 (2006).

[25] S. Hofmann, G. Csányi, A. C. Ferrari, M. C. Payne, and J. Robertson, Physical Review Letters 95, $036101(2005)$.

[26] X. Fan, R. Buczko, A. A. Puretzky, D. B. Geohegan, J. Y. Howe, S. T. Pantelides, and S. J. Pennycook, Physical Review Letters 90, 145501 (2003).

[27] Q.-M. Zhang, J. C. Wells, X. G. Gong, and Z. Y. Zhang, Physical Review B 69, 205413 (2004).

[28] S. Reich, L. Li, and J. Robertson, Chemical Physics Letters 421, 469 (2006).

[29] P. Larsson, J. A. Larsson, R. Ahuja, F. Ding, B. I. Yakobson, H. Duan, A. Rosén, and K. Bolton, Physical Review B 75, 115419 (2007).

[30] F. Ding, P. Larsson, J. Larsson, R. Ahuja, H. Duan, A. Rosen, and K. Bolton, Nano Letters 8, 463 (2008).

[31] H. Amara, C. Bichara, and F. Ducastelle, Physical Review B 73, 113404 (2006).

[32] H. Amara, C. Bichara, and F. Ducastelle, Physical Review Letters 100, 056105 (2008).

[33] A. Borjesson, W. Zhu, H. Amara, C. Bichara, and K. Bolton, Nano Letters 9, 1117 (2009).

[34] Y. Ohta, Y. Okamoto, S. Irle, and K. Morokuma, Carbon 47, 1270 (2009).

[35] Y. Shibuta and S. Maruyama, Chemical Physics Letters 382, 381 (2003).

[36] J. Zhao, A. Martinez-Limia, and P. B. Balbuena, Nanotechnology 16, 575 (2005).

[37] F. Ding and K. Bolton, Nanotechnology 17, 543 (2006).

[38] V. Vitale, A. Curioni, and W. Andreoni, J. AM. CHEM. SOC. 130, 5848 (2008).

[39] G. Kresse and J. Furthmüller, Phys. Rev. B 54, 11169 (1996).

[40] J. P. Perdew, J. A. Chevary, S. H. Vosko, K. A. Jackson, M. R. Pederson, D. J. Singh, and C. Fiolhais, Phys. Rev. B 46, 6671 (1992).

[41] W. Zhu, A. Börjesson, and K. Bolton, Carbon 48, 470 (2010).

[42] J. J. Mortensen, L. B. Hansen, and K. W. Jacobsen, Phys. Rev. B 71, 035109 (2005).

[43] J. P. Perdew, K. Burke, and M. Ernzerhof, Phys. Rev. Lett. 77, 3865 (1996).

[44] S. R. Bahn and K. W. Jacobsen, Comput. Sci. Eng. 4, 56 (2002).

[45] A. G. Nasibulin, P. V. Pikhitsa, H. Jiang, and E. I. Kauppinen, Carbon 43, 2251 (2005).

[46] C. Cheung, A. Kurtz, H. Park, and C. Lieber, Journal of Physical Chemistry B 106, 2429 (2002).

[47] A. K. Schaper, H. Hou, A. Greiner, and F. Phillipp, Journal of Catalysis 222, 250 (2004), 
ISSN 0021-9517.

(a)

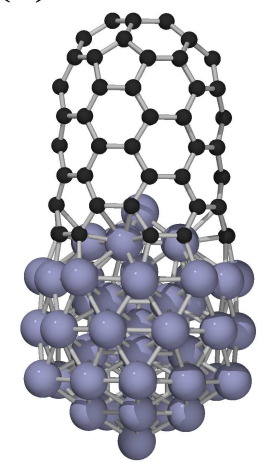

$-968.58 \mathrm{eV}$

(c)

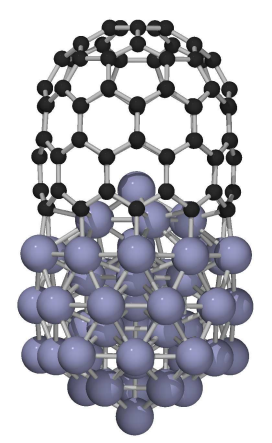

$-970.88 \mathrm{eV}$ (b)

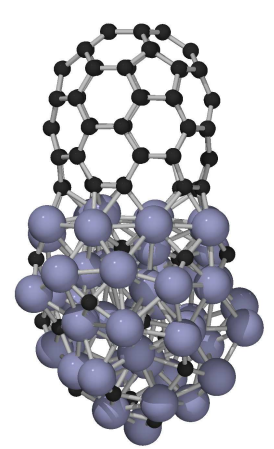

$-960.39 \mathrm{eV}$

(d)

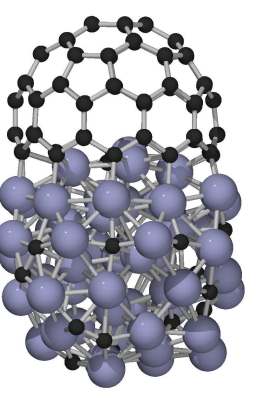

$-960.85 \mathrm{eV}$

FIG. 1: Typical minimum energy structures of capped CNTs attached to $\mathrm{Ni}_{55}$ and $\mathrm{Ni}_{55} \mathrm{C}_{20}$ clusters. The CNT chiralities are $(5,5)((\mathrm{a})$ and $(\mathrm{b}))$ and $(10,0)((\mathrm{c})$ and $(\mathrm{d}))$ and the total energy of the structures are given below the structure. 
CNTs attached to pure $\mathrm{Ni}$

\begin{tabular}{|l|l|l|l|l|}
\hline System & $E_{\text {tot }}$ & $E_{C N T}$ & $E_{\text {cluster }}$ & $E_{\text {adhesion }}$ \\
\hline CNT (3,3) (VASP) & $-387,44$ & $-326,98$ & $-50,06$ & $-10,40$ \\
CNT (5,5) (VASP) & $-968,58$ & $-701,75$ & $-250,61$ & $-16,22$ \\
CNT (9,1) (VASP) & $-968,71$ & $-695,45$ & $-250,61$ & $-22,65$ \\
CNT (10,0) (VASP) & $-970,88$ & $-695,08$ & $-250,61$ & $-25,20$ \\
CNT (3,3) (GPAW) & $-420,20$ & $-328,58$ & $-81,02$ & $-10,60$ \\
CNT (10,0) (GPAW) & $-1104,69$ & $-696,06$ & $-381,30$ & $-27,33$ \\
\hline
\end{tabular}

CNTs attached to Ni carbides

\begin{tabular}{|l|l|l|l|l|}
\hline System & $E_{\text {tot }}$ & $E_{C N T}$ & $E_{\text {cluster }}$ & $E_{\text {adhesion }}$ \\
\hline CNT (3,3) (VASP) & $-385,66$ & $-274,53$ & $-100,71$ & $-10,38$ \\
CNT (5,5) (VASP) & $-960,39$ & $-520,01$ & $-426,48$ & $-13,90$ \\
CNT (9,1) (VASP) & $-959,72$ & $-513,26$ & $-426,48$ & $-19,98$ \\
CNT (10,0)(VASP) & $-960,85$ & $-513,07$ & $-426,48$ & $-21,30$ \\
CNT (3,3) (GPAW) & $-418,49$ & $-276,06$ & $-132,62$ & $-9,81$ \\
CNT (10,0) (GPAW) & -1097.03 & $-513,19$ & $-559,68$ & $-24,16$ \\
\hline
\end{tabular}

TABLE I: Minimum energies $(\mathrm{eV})$ for $(3,3),(5,5),(9,1)$, and $(10,0)$ CNTs attached to pure $\mathrm{Ni}_{13}$ and $\mathrm{Ni}_{55}$ clusters (top table) and nanotubes with the same chiralities attached to $\mathrm{Ni}_{13} \mathrm{C}_{6}$ and $\mathrm{Ni}_{55} \mathrm{C}_{20}$ clusters (bottom table). The minimum energies are also given for the individual parts of the system, i.e., the CNTs and metal (carbide) clusters. The data obtained by VASP (PW91) and GPAW (PBE) have been explicitly indicated. 
CNT attached to pure $\mathrm{Ni}$

\begin{tabular}{|l|l|l|l|l|}
\hline System & $E_{\text {tot }}$ & $E_{C N T}$ & $E_{\text {cluster }}$ & $E_{\text {adhesion }}$ \\
\hline CNT (5,5) & $-960,39$ & $-514,96$ & $-421,37$ & $-24,07$ \\
CNT (9,1) & $-959,72$ & $-509,83$ & $-419,94$ & $-29,95$ \\
CNT (10,0) & $-960,85$ & $-509,38$ & $-421,27$ & $-30,2$ \\
\hline
\end{tabular}

TABLE II: Minimum energies $(\mathrm{eV})$ for $(5,5),(9,1)$, and $(10,0)$ CNTs with 60 atoms attached to $\mathrm{Ni}_{55} \mathrm{C}_{20}$. The energies given for the individual parts of the system, i.e., the CNTs and $\mathrm{Ni}_{55} \mathrm{C}_{20}$ clusters, as well the direct adhesion energy were obtained after a direct separation of the CNT$\mathrm{Ni}_{55} \mathrm{C}_{20}$ system, i.e., no relaxation of the individual systems were performed.

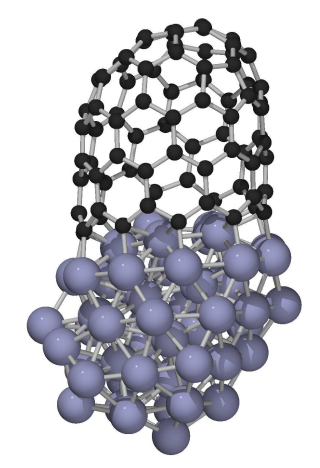

$-965.76 \mathrm{eV}$

FIG. 2: A typical minimum energy structure of a $(9,1)$ CNT attached to an amorphous $\mathrm{Ni}_{55}$ cluster. The total energy of this structure is approximately $3 \mathrm{eV}$ higher than for the corresponding system with an icosahedral $\mathrm{Ni}_{55}$ cluster. 
(a)

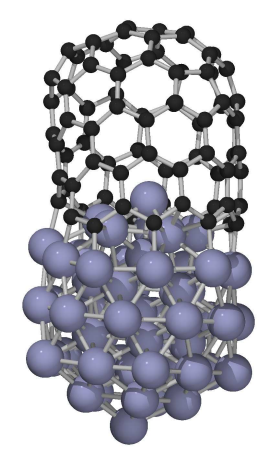

$-968.71 \mathrm{eV}$

(c)

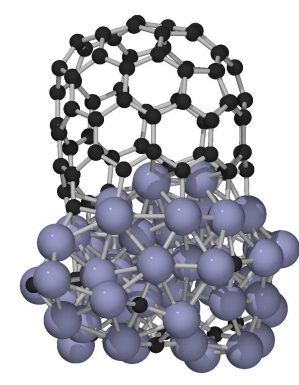

$-963.50 \mathrm{eV}$ (b)

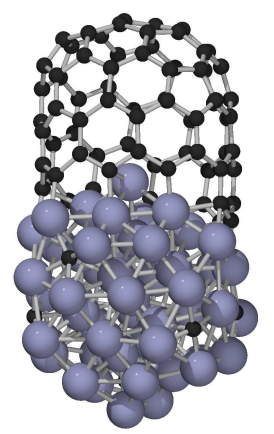

$-965.16 \mathrm{eV}$

(d)

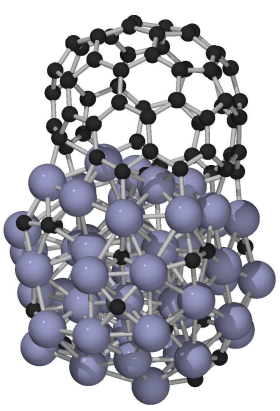

$-959.72 \mathrm{eV}$

FIG. 3: Typical minimum energy structures of a capped $(9,1) \mathrm{CNT}$ attached to a $\mathrm{Ni}_{55} \mathrm{C}_{X}$ cluster. The number of carbon atoms, $X$, in the cluster is 0 (a), 6 (b), 14 (c) and 20 (d). The total energy of the system increases with increased carbon concentration in the cluster. 


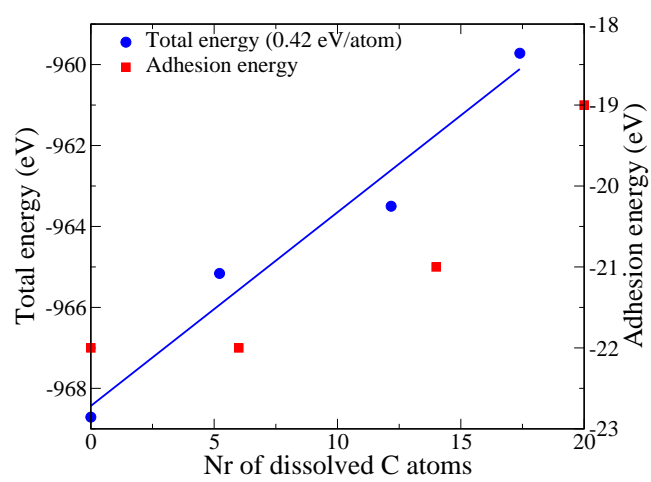

FIG. 4: Total energies (round symbols) and adhesion energies (square symbols) of a $(9,1)$ CNT attached to a $\mathrm{Ni}_{55} \mathrm{C}_{X}$ cluster as a function of the number of $\mathrm{C}$ atoms dissolved in the cluster. The solid line is a linear fit to the total energy and the slope of the linear fit is seen in the legend.

A $(9,1)$ CNT attached to pure Ni clusters

\begin{tabular}{|l|l|l|l|l|}
\hline Time & $E_{\text {tot }}$ & $E_{C N T}$ & $E_{\text {cluster }}$ & $E_{\text {adhesion }}$ \\
\hline 300 & $-954,49$ & $-683,54$ & $-243,83$ & $-27,12$ \\
600 & $-953,18$ & $-682,80$ & $-242,30$ & $-28,08$ \\
900 & $-953,81$ & $-682,82$ & $-244,05$ & $-26,94$ \\
1200 & $-953,72$ & $-682,38$ & $-242,57$ & $-28,77$ \\
\hline
\end{tabular}

A $(9,1)$ CNT attached to Ni-carbide clusters

\begin{tabular}{|l|l|l|l|l|}
\hline Time & $E_{\text {tot }}$ & $E_{C N T}$ & $E_{\text {cluster }}$ & $E_{\text {adhesion }}$ \\
\hline 300 & $-944,02$ & $-504,67$ & $-412,30$ & $-27,05$ \\
600 & $-944,10$ & $-505,32$ & $-412,34$ & $-26,44$ \\
900 & $-942,87$ & $-504,48$ & $-411,72$ & $-26,67$ \\
1200 & $-944,41$ & $-502,70$ & $-413,53$ & $-28,18$ \\
\hline
\end{tabular}

TABLE III: The total energies of $(9,1)$ CNT attached to pure Ni clusters (top) and Ni-carbide clusters (bottom) as well as the total energies of the individual constituents and the direct adhesion energy. The CNT-cluster system had been subjected to MD simulation and the systems were sampled after $300 \mathrm{fs}, 600 \mathrm{fs}, 900 \mathrm{fs}$ and $1200 \mathrm{fs}$. 